\title{
Applications of Polarized Deuteron Beams for Studies of Few-Nucleon Dynamics in d-p Breakup.
}

I. Ciepał, ${ }^{a}$ I. Skwira-Chalot, ${ }^{b}$ N. Kalantar-Nayestanaki, ${ }^{c}$ G. Khatri, ${ }^{d}$ B. Kłos, ${ }^{e}$ St. Kistryn, ${ }^{d}$ A. Kozela, ${ }^{a}$ P. Kulessa, ${ }^{a}$ J. Messchendorp, ${ }^{c}$ W. Parol, ${ }^{d}$ E. Stephan, ${ }^{e}$ B. Włoch ${ }^{f}$

${ }^{a}$ Institute of Nuclear Physics PAS, 31-342, Kraków, Poland

${ }^{b}$ Faculty of Physics, University of Warsaw, 02-093, Warsaw, Poland

${ }^{c}$ KVI-CART, University of Groningen, NL-9747 AA Groningen, The Netherlands

${ }^{d}$ Institute of Physics, Jagiellonian University, 30-059, Kraków, Poland

${ }^{e}$ Institute of Physics, University of Silesia, 40-007, Katowice, Poland

${ }^{f}$ AGH - University of Science and Technology, Faculty of Physics and Applied Computer Science,

PL-30059 Kraków, Poland

\begin{abstract}
A large set of high precision vector and tensor analyzing power data of ${ }^{1} \mathrm{H}(\vec{d}, p p) n$ breakup reaction was obtained at energy of $130 \mathrm{MeV}$. Studies of the breakup process with the use of polarized beams provide basis for thorough tests of the nuclear force structure. Such observables are sensitive to spin-dependent part of the interaction, what makes them interesting for testing theoretical calculations based on various approaches modeling the interaction in few-nucleon systems. The data presented as a function of a new set of invariants, introduced to describe the process with three nucleons in the final state, were confronted with the set of the modern calculations.
\end{abstract}

XVIth International Workshop in Polarized Sources, Targets, and Polarimetry, PSTP2015,

14-18 September 2015,

Bochum, Germany 


\section{Introduction}

Deuteron breakup in collision with a proton, leading to a final state of three-nucleon $(3 \mathrm{~N})$ continuum is one of the simplest precesses to study dynamics of few nucleons. The process is characterized by a rich kinematics of the final state what makes it selective regarding the applied model of interaction. Experiments with polarized targets or beams give access to a large number of observables, which are sensitive to the dynamical ingredients, which are hidden in the unpolarized case, averaged over spin states. The polarization observables, e.g. the analyzing powers, are sensitive to spin-dependent part of the interaction, what makes them interesting for testing theoretical calculations based on various approaches describing the interaction in few-nucleon systems.

In a low and medium energy domains the properties of few-nucleon systems are successfully modeled with the use of the realistic nucleon-nucleon (NN) potentials, coupled-channel (CC) calculations with realistic potential including non-nucleonic degrees of freedom [1] or Chiral Perturbation Theory (ChPT) [2]. At intermediate energies, effects of the few-nucleon dynamics beyond the pairwise NN interaction start to play the role. Due to exact theoretical calculations and high level of experimental precision, it is possible to study very subtle effects, for example Three-Nucleon Force (3NF) [3]. In order to correctly describe the system dynamics calculations must include the model of 3NF (e.g. Tucson Melbourne TM force [4]) and/or the Coulomb force [5]. The experimental studies of the breakup process allow one to test the nuclear force structure with possible feedback on the force models.

In the present work extensive data sets obtained in a series of experiments are used to investigate the effects of the Coulomb force and $3 \mathrm{NF}$ on the polarization observables. The comparison between the data and the theory is performed for observables presented as function of variables based on Lorentz-invariants.

\section{Experiments}

In the experiments the polarized deuteron beams were produced with the use of the ion sources of the COSY (IKP FZ-Jülich, Germany) and AGOR (KVI Groningen, The Netherlands) accelerators.

The measurement at FZ-Jülich was conducted with the Germanium Wall (GeWall) setup [6] at the deuteron beam energy of $130 \mathrm{MeV}$. The beam has been produced in the polarized ion source [7] in two states: transversally vector polarized $\left(P_{z}, P_{z z}\right)=\left(-\frac{2}{3}, 0\right)$ and unpolarized $\left(P_{z}, P_{z z}\right)=(0,0)$. GeWall consisted of three high-purity germanium position sensitive detectors. Two different types of the detectors were used: a thin transmission detector Quirl with a high spatial resolution for determining the position and energy loss ( $\Delta E$ detector) of the passing charged particles, and two thick detectors $E 1$ and $E 2$ for measurement of particle energies with an excellent resolution. The angular acceptance of the apparatus was $5^{\circ}-14^{\circ}$ for the polar and $2 \pi$ for the azimuthal angles.

With the SALAD detector (KVI Groningen) [8] and the deuteron beam energy of $130 \mathrm{MeV}$ a rich set of vector and tensor analyzing powers data was obtained. The polarized beam was produced in polarized ion source POLIS in six polarization states: $\left(P_{z}, P_{z z}\right)=\left(+\frac{1}{3},+1\right),\left(+\frac{1}{3},-1\right),(0$, $+1),(0,-2),\left(+\frac{2}{3}, 0\right),\left(-\frac{2}{3}, 0\right)$. The detector system was composed of a two layers of scintilla- 
tor hodoscope: $\Delta E$ (24 strips) and $E$ (24 slabs) and multiwire proportional chamber (MWPC) for momenta reconstruction. The detector covered the angular range from $15^{\circ}$ to $37^{\circ}$.

\section{Results and conclusions}

New generation measurements of the ${ }^{1} \mathrm{H}(\vec{d}, p p) n$ breakup reactions in a wide phase-space region provided a very rich set of vector $A_{x}, A_{y}$ and tensor $A_{x x}, A_{x y}, A_{y y}$ analyzing powers. Such extended database can be used for systematic investigations of the $3 \mathrm{~N}$ system dynamics. The experimental investigations at FZ-Jülich [6,9] were focused on a very forward part of the phase space. The studies in this angular range showed that the electromagnetic interaction, as well as the other dynamical effects connected with the $3 \mathrm{~N}$ dynamics, do not influence the vector analyzing-power data [6]. This finding is opposite to that observed in the cross section [9]. In view of this finding, the further discussion is based on the data obtained with the SALAD detector only.

In the previous studies comparisons between the data and theory were performed in terms of standard variables which define a specific configuration of the two detected breakup protons, i.e. geometry decribed by combinations of their polar angles, their relative azimuthal angle and an energy expressed in terms of arc-length along the given kinematics. Such representation is inadequate and not practical when one needs to make a cross analysis over data collected in wide energy and phase-space ranges. Therefore, an alternative way of the description of few-body reaction kinematics was proposed in [10]. Mandelstam variables for a two-body reaction were redefined to describe three-body final states. One of the variables, the kinetic energy of the relative motion of the two breakup protons $\left(E_{r e l}\right)$, was chosen to study the effects of the Coulomb force and $3 \mathrm{NF}$ in the $A_{x}$ and $A_{y y}$ analyzing-power data. To search for the electromagnetic force effects the calculations based on the Argonne V18 (AV18) potential combined with Urbana IX 3NF (UIX) with (AV18+UIX+C) and without (AV18+UIX) Coulomb interaction included were analysed [5]. In the case of the $3 \mathrm{NF}$ effects, the predictions based on the set of the two-nucleon $(2 \mathrm{~N})$ realistic potentials (Argonne V18, Nijmegen I, II and CD-Bonn) were confronted with the same calculations including Tucson Melbourne (TM) $3 \mathrm{NF}(2 \mathrm{~N}+\mathrm{TM})$ [4]. The predictions obtained with the $2 \mathrm{~N}$ potentials were treated altogether creating a band. A center of the band was then used for further comparisons with the data. In a similar way the $2 \mathrm{~N}+\mathrm{TM}$ calculations were treated. Since the bands are relatively narrow (as compared to the studied effects) such an approach is justified. To compare theory with the experimental results first the calculations were corrected over the detector acceptance and the following differences were obtained:

a) $A_{i}$ (theory+effect) $-A_{i}$ (theory)

b) $A_{i}$ (data) $-A_{i}$ (theory)

c) $A_{i}$ (data) $-A_{i}$ (theory+effect)

where $A_{i}$ denotes a given analyzing power, theory denotes $2 \mathrm{~N}$ or AV18+UIX calculations, effect is a given dynamical component i.e. Coulomb force (see Fig. 四) or TM 3NF (see Fig. \) added to that theory and data refers to the experimental results.

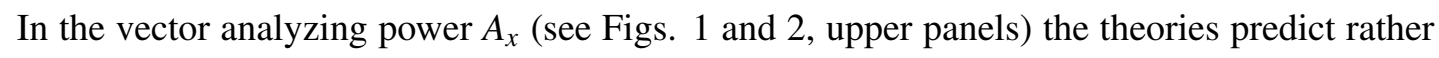
small effects of the Coulomb force and $3 \mathrm{NF}$ and the data seem to confirm the calculations. In case 

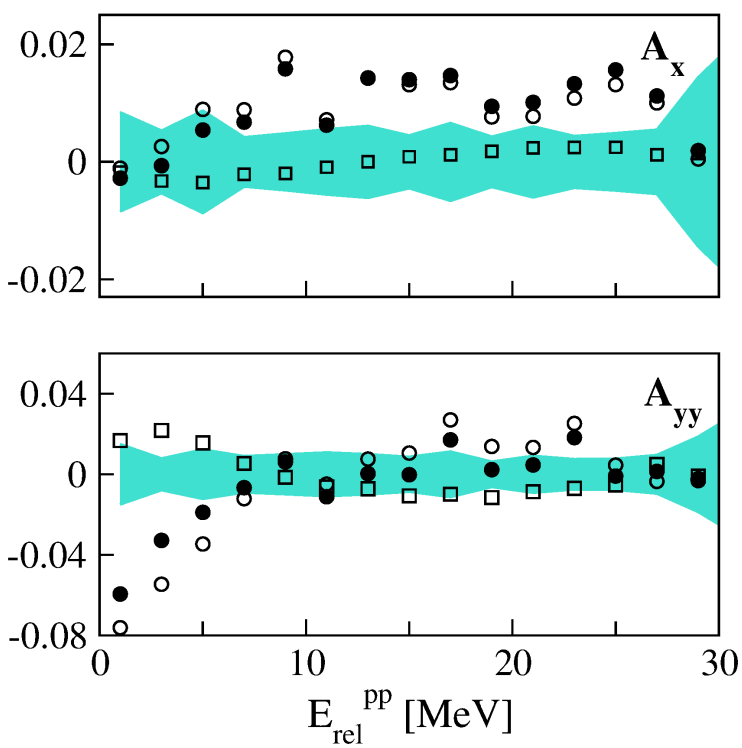

Figure 1: (Color online) Net effects of the Coulomb force in the $A_{x}$ and $A_{y y}$ analyzing powers of the $d p$ breakup at $130 \mathrm{MeV}$. The data are presented as a function of the relative energy of the two breakup protons. The y-coordinate represents the differences between: data and AV18+UIX calculations (full dots), data and AV18+UIX+C theory (circles). Empty squares represent the difference between both of the calculations. The turquoise (grey) band shows the statistical uncertainties.
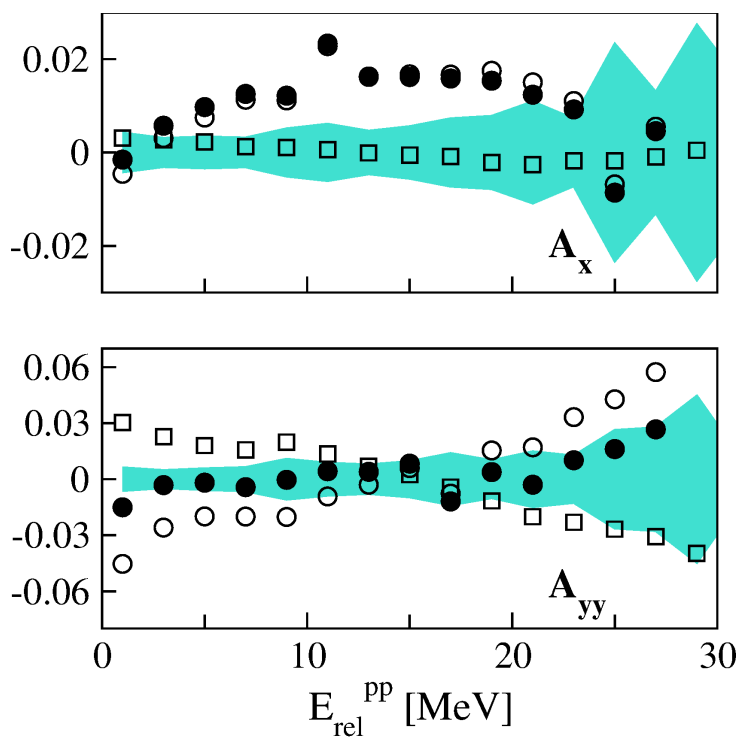

Figure 2: (Color online) The same as Fig.1, but for 2N and 2N+TM 3NF predictions. The y-coordinate represents the differences between: data and $2 \mathrm{~N}$ calculations (full dots), data and 2N+TM $3 \mathrm{NF}$ theory (circles). Empty squares represent the difference between both of the calculations.

of the tensor $A_{y y}$ analyzing power (see Figs. 凹 and 凤, lower panels) the theories predict significant effects of the Coulomb force at small $E_{r e l}$ and rather small for the higher values. Such behavior is consistent with the similar considerations for the cross-section data [9-11]. One can conclude that 
the data are well reproduced by $2 \mathrm{~N}$ calculations in the whole studied phase space.

The predicted effects of $3 \mathrm{NF}$ in the tensor analyzing power are quite large. However, the data do not confirm this aspect of the calculations. The effects of TM 3NF are opposite in comparison with influences observed when the data are related to the theories, see Fig. \} \text { , lower panel. In } these predictions there is still room for the Coulomb or/and relativistic components. The previous findings [10] for the AV18+UIX+C model, which contain all the dynamical ingredients except relativity, showed that adding the Coulomb force do not reduce the discrepancies between the data and theory. This suggests the existence of some missing ingredients in the spin part of the $3 \mathrm{NF}$ model, either relativistic effects or unresolved problems in our understanding of the $3 \mathrm{NF}$ structure. What is more, magnitude of the effects predicted by the theoretical calculations is not contained within the band reflecting the experimental uncertainties.

Besides $E_{\text {rel }}$ which corresponds to the invariant mass of the protons emerging from the breakup reaction, the 4-momentum transfer variables can also be used to study the system dynamics. There are four independent variables, so one can investigate also e.g. two-dimensional correlations between them to search for other aspects of the $3 \mathrm{~N}$ dynamics.

\section{Outlook}

Precise and systematic studies of the breakup reaction in a large part of the phase space $[10,12]$ are very important for understanding of the interaction between nucleons in few-nucleon systems. Currently available theoretical approaches to describe the interaction need very precise and large experimental database to be verified and further developed. To perform cross-energy systematic comparisons of the predictions and the data the invariant coordinates were introduced. They constitute a convenient basis for reaching more general conclusions.

New experiments to study $3 \mathrm{~N}$ system dynamics in the four-body environment are planned. Such investigations, utilizing a polarized ${ }^{3} \mathrm{He}$ target, are planned at the Cyclotron Center Bronowice of the Institute of Nuclear Physics in Cracow. An experimental apparatus which will be used is already under construction. The studies will allow one to fill a data-deficient sector of $4 \mathrm{~N}$ systems.

This work was supported by the Polish 2013-2016 science found as research Project No. 2012/05/E/ST2/02313.

\section{References}

[1] A. Deltuva, R. Machleidt, P.U. Sauer, Phys. Rev. C 68, 024005 (2003).

[2] E. Epelbaum, Prog. Part. Nucl. Phys. 57, 645 (2006).

[3] H. Witała et al., Phys. Rev. C 83, 044001 (2011).

[4] S.A. Coon and H.K. Han, Few-Body Syst. 30, 131 (2001).

[5] A. Deltuva et al., Phys. Rev. C 80, 064002 (2009).

[6] I. Ciepał at al., Phys. Rev. C 85, 017001 (2012).

[7] R. Weidmann at al., Rev. Sci. Instrum. 67, 1357 (1996). 
[8] E. Stephan et al., Phys. Rev. C 76, 057001 (2007).

[9] I. Ciepał et al., Few-Body Syst. 56, 665 (2015).

[10] St. Kistryn, E. Stephan, J. Phys. G: Nucl. Part. Phys. 40, 063101 (2013).

[11] St. Kistryn et al., Phys. Lett. B 641, 23-27 (2006).

[12] N. Kalantar-Nayestanaki et al., Rep. Prog. Phys. 75, 016301 (2012). 